\title{
Cockpit/cabin Crew Communication: Problems and Countermeasures
}

\author{
Shou Xi Zhu, a ${ }^{1,}$ and Wen Lai Ma ${ }^{2, b}$ \\ ${ }^{1}$ Flying College, Binzhou University, Binzhou, China \\ ${ }^{2}$ Flying College, Binzhou University, Binzhou, China \\ azhushouxi@163.com, bmawenlai@163.com
}

\begin{abstract}
Keywords: Communication; Crew resource management; Barrier; Cabin crew; Collaboration.
\end{abstract}
\begin{abstract}
Communication and collaboration between the cockpit and cabin is one of the important human factors affect flight safety and it is an important part of crew resource management. In the paper, in order to better facilitate communication and collaboration between cockpit and cabin, we analyzed the communication barriers between the cockpit and cabin from the aspects such as history, nature of work, culture and so on. Then analyzed the various sources of information required that the cabin crew communicate with the cockpit crew, such as passengers, standard operation procedures, Cabin Intercommunication Data System, catering staff, maintenance staff, etc., studied the methods to obtain information from the information sources. Finally, this paper presented some ways to promote the communication between the cockpit crew and cabin crew, including briefing, good information sharing and good crew coordination training. This study can effectively promote the effect of crew resource management and is of great significance to flight safety.
\end{abstract}

\section{Introduction}

In recent years, some aviation accidents and incidents had proven to be caused by bad communication and inadequate co-ordination between the cockpit and cabin crew [1]. Crew resource management (CRM) is an important measure to ensure flight safety. The FAA promotes the development of the CRM concept through the extensive research, experiences that airlines implement CRM projects and the lessons obtained. In the modification of FAA AC120-51, CRM gets a new definition and the advisory circular gives the latest progress of CRM. In this advisory circular, CRM contains all the personnel related to flight, including cockpit crew, cabin crew, air traffic controllers, engineering maintenance staff and so on [2]. The meaning of CRM is from cockpit resource management to crew resource management. The replacement of the term shows an important stage of CRM development. As the crew members of an airplane, collaboration between the cockpit crew and cabin crew is very important for flight safety although they are two different units for training, scheduling and are considered to be two independent crew [3]. However, the reality is that many airlines do not lay emphasis on the communication and collaboration between the cockpit crew and cabin crew. Although some airlines consider that they should be pay attention to the synergy of cockpit and cabin, but the role of cabin crew in flight safety only limited to maintain the order of the cabin and organize safe evacuation. In training, except the critical situation, emergency evacuation or air hijacked, there is little emphasis on communication between the cockpit and cabin, which brought a lot of hidden trouble to the flight safety and cannot conform to the requirements of CRM.

\section{Barriers of Cockpit/cabin Crew Communication}

Cabin crew members should play the role of extended function of the cockpit crew to ensure flight safety [4]. Cockpit crew and cabin crew should play a team function to achieve the shared safety goals. According to the requirements of CRM, once any situation that possible threat to flight safety occurs, such as feelings, words and actions, the cabin crew members must be vigilant and should report to the chief attendant and cockpit crew immediately. However, in fact, the communication between the cockpit crew and cabin crew is relative weakness. Tradition affects the modern crew to a certain extent. The early pilots were relatively brave and advocated personal technology, in contrast, early 
flight attendants were nurses and the selection criteria were obedience and subordination. The early cockpit and cabin crew communication used the army and navy's command chain [5], which was traditional and stereotype. In management, the pilots and flight attendants were classified as a separate department. At the same time, the crew members were asked not to communicate in early flight manual. Today, these guidelines are still influencing the crew communication to some extent.

The communication between cockpit crew and cabin crew is shown in figure 1 .

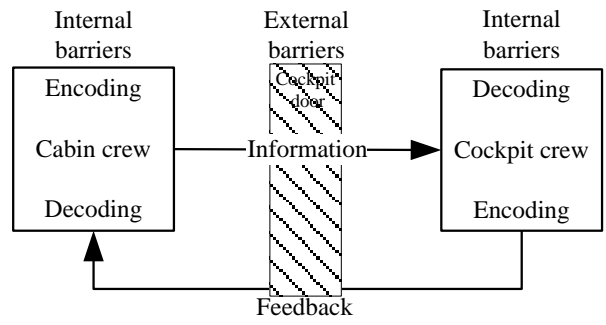

Fig. 1 Communication between cockpit crew and cabin crew

There are many factors called barriers that can affect the communication between the cockpit crew and cabin crew [6], external barriers such as light, noise, resource location, work load, etc. Internal barriers such as attitude, personality, concept, cultural differences and so on. In addition, there are also some specific reasons, which are related to the work characteristics, including gender differences, social psychological differences and poor receptivity to each other's job responsibilities. For they belonging to different departments and separated to two different areas of the airplane by the enhanced cockpit doors, that makes they feel there are two crews on the airplane. At the same time, the duty arrangement and working time are different. Most cockpit crew members are men, they think their career is lifelong, but most cabin crew members are women, most of them consider their career is temporary. Besides, for the quiet cockpit principle, the cockpit crew will do not know how and when to contact the cockpit.

Communication is of great importance for a safe flight. The poor communication has induced many security problems in the aviation industry. Among them, part of the security problems is due to the inappropriate cockpit/cabin crew communication, which we need to attract attention [7]. Pilots and flight attendants have a same goal, which is the highest priority of flight safety. Most crew has good teamwork and communication between cockpit crew and cabin crew is smooth and accurate. Nonetheless, when there is a communication barrier or even non-communication, it is important to know how this will endanger the flight safety. Many accident analysis shows that the communication of information between the cabin and the cockpit also plays a key role for flight safety.

\section{Information Source for Cockpit/cabin Crew Communication}

An important aspect of crew resource management is to consider all available resources [8]. There are many information sources available on the plane that can allow the crew to detect, judge the situation and take effective steps. A lot of information that the pilots obtain is from the cabin crew which depending on the effective use of the information source.

Passengers. Passengers on the airplane are the largest source of information. Sometimes passengers are the first to provide information and cause crew members pay attention to the abnormal condition, for example, abnormal odours or abnormal sound. Cabin crew must pay attention to any statements of passengers' information on the following matters:

- Cabin interior (noise, gas, smoke, fire, loose objects, etc.);

- Outside the aircraft (wing, fuselage, etc.);

- External environment (runway, weather, etc.);

- Abnormal behavior of other passengers (fiddle with abnormal objects, etc.).

Cabin crew members should take account of any passenger report on unusual circumstances, and notify chief attendant and cockpit crew in time. Passengers should be encouraged to communicate with cabin crew, such as adding the following phrase in the passenger briefing: "If you find any 
security problems during the flight, please remind the crew members, and you will get a small gift, thank you!" Such phrases can encourage passengers to remind the crew and express their concerns.

Standard Operation Procedure (SOP). SOP provides a common understanding basis for the entire crew [9]. Crew members may have a variety of different cultures and nationalities. Then the standard operation procedure can become a common language that can provide an environment for these crew members to work together and communication. In a sense, the standard operation procedure is one of the most reliable communication forms for crew.

Cabin Intercommunication Data System (CIDS). CIDS is an automated information system for the cockpit crew, cabin crew and ground staff. CIDS operating, controlling and monitoring of the main cabin systems such as air conditioner, communication, fire protection, de-icing, lighting, waste and water, etc. CIDS system can also automatically detect failure of its components and connected equipment. If a fault is detected, it will display an alert on the flight attendant panel to alert the crew.

Communication with Maintenance Staff. Aircraft maintenance personnel are a critical part of daily operation. Aircraft maintenance personnel bear a heavy workload, especially during a short stop, the maintenance work must be completed within the time limit.

The communication with maintenance personnel is also important, through the communication, the cabin crew can understand any technology failure impact to plane. For example, a reserved device, a device does not work, an open breaker, or any device that may affect the flight. When cabin crew communicates with maintenance personnel, particularly record on the cabin maintenance logbook, they should use clear terminology to report failure and the measures taken, and clearly define the location and description of the fault.

Ensure the cabin maintenance logbook is complete and list all the maintenance equipment before the plane arrival. Maintenance personnel are also an important part of flight safety chain and play a key role in the daily operation. When communicate with maintenance personnel, the cabin crew members should express their courtesy and respect, and note the following issues:

- Verbal communication between the chief attendant and maintenance personnel is equally important;

- Ensure that the cabin crew members understand the influence of any technical malfunctions, and their influence on cabin service may cause;

- Use clear terminology to report failure and the measures taken;

- If possible, report the fault to the most appropriate personnel.

Communication and Cooperation with Catering Staff. Due to catering staff may not understand the influence of their actions on security, so when the catering staff boarding, there should be a cabin crew member in the kitchen area to monitor catering operations. When monitoring catering, the cabin crew member should ensure that:

- Small trolleys and their brakes work effectively;

- Completely check the oven if there are any unusual objects, papers, towels, etc.;

- The handle and lock of storage boxes should be in the work instructions.

Communication and Cooperation with Check-in Staff. The passengers boarding need close cooperation of cockpit crew, cabin crew and ground staff. The priority of check-in staff is to make all passengers boarding to ensure "take off on time". However, sometimes due to the late of aircraft arrival into airport, the ground staff will be responsible for all the passengers, and is responsible for the possibilities that passengers may miss a connecting flight. In order to avoid any problems before the completion of cabin ready and issues affecting passengers boarding, cabin crew members should contact the check-in staff initiative, such as notify the cabin status to check-in staff and ensure that the passengers will not boarding before the cockpit crew and cabin crew meeting.

The above information is from the different departments of airline, so the airline should formulate corresponding training plan to ensure the effective use of the above information.

\section{Effective Measures to Promote the Cockpit/cabin Crew Communication}

Introduction and Briefing. At the beginning of each flight, the cockpit crew should organize a briefing with cabin crew to explain the details of the work and the requirements for inspection and to 
cooperate with each other. Briefing is an integral part of the most important tasks in flight preparation [10]. Sometimes, the crew members did not recognize each other, but they need to collaborate closely, and sometimes, this situation needs to continue for a long period. The briefing is a starting point to establish a harmonious relationship, and needs to encourage open, friendly, mutual respect and professional attitude. A good briefing has the following features: appropriate, summary, clear and concise.

The briefing should be appropriate and summary, it should include all key points. Briefing is the activity to organize crew. Its task is to arrange the working position and the safe responsibility of each crew member. All crew members should learn briefing. It is an important way to establish a shared working environment and the expected flight for cockpit crew and cabin crew, and also an opportunity to promote two-way communication for cockpit crew and cabin crew.

Briefing can help to create a favorable working environment for safe flight, when undertaking a briefing, we should pay attention to the following issues:

- First, crew members should introduce themselves friendly;

- Use a professional and friendly language;

- Mutual respect;

- With safety awareness;

- Cabin crew members should be encouraged to report any threat that they feel to flight safety to the chief attendant and cockpit crew;

- Discuss the "quiet cockpit" rule with pilots, in order to define an acceptable factor to contact the flight crew at this time;

- Understanding of each other's workload.

Briefing is of great importance to each flight operation. It can create a tone of communication that helps to flight safety. Through briefing, the crew can achieve the following goals:

- Establish a common goal;

- Exchange information and improve the situational awareness;

- Mutual monitoring the performance of activities;

- Good situation report;

- Establish friendly and professional atmosphere.

It should be emphasized again that the cabin crew and cockpit crew must implement the briefing jointly. If the briefing cannot be executed before boarding, then the crew should make every effort to communicate before takeoff. When communication, the cockpit crew and the chief attendant should encourage all the crew members to communicate openly. Communication is two-way and listen is also an important part of it. Listen is an important communication skill. Active listening will make you focus directly on the message expressed by the speakers and can get more information when you listen to other person's speech. At the same time, good listening is also easier to get positive feedback, a better exchange of information and a better communication effect.

Information Sharing. Effective communication is more complex, some factors such as tone, expression, gesture, etc., will influence others to receive information. In most situations, the communication between cockpit and crew is through interphone. This way of communication cannot provide visual feedback, so the expression and tone are more important. The information shared in communication should be real and include all critical information points. When sending information, crew members who providing information should clearly express in order to be heard and understood. Keep the information concise so that the person who receiving information has the opportunity to ask questions to clarify information. When receiving the information, the answer should be clear, concise and realistic. After receiving the information, the receiver should clarify important information to the information provider actively. The method is to interpret the information and encourage feedback in order to ensure that the information is properly understood.

Joint Training. Strengthen joint training between the cockpit and cabin. First, enhance flight attendants report skills about the cabin security, make them effective and active communicate cabin security problems with cockpit crew. In addition, provide a common communication platform for cockpit crew and cabin crew. Training in a simulator cockpit which cockpit doors is open or in a 
classroom, in order to avoid estrangement caused by the cockpit doors. This may help to form a good and equality communication atmosphere, enhance mutual understanding and build security and positive group atmosphere.

\section{Conclusion}

Good cockpit/cabin crew communication is the key element to ensure a flight mission that performs smooth and safe. A good briefing can be able to create a good team performance, we should use some briefings to encourage communication, promote team work and establish a harmonious relation between crew members. Standard operation procedures are the common language in the airplane, which ensures that all crew members can be familiar with the flight standards and expectations. Take full advantage of the automation systems and play an active role with CIDS. Make full use of the information source, communicate and cooperate with the other crew members, maintenance personnel, catering staff and check-in personnel, etc. Communicate with passengers and make them feel comfortable and willing to interact with the crew members.

\section{Acknowledgement}

This research was financially supported by scientific research project of Binzhou University (Grant NO.BZXYG1316, BZXYG1320).

\section{References}

[1] Yun Xiao, Analysis of Communication Obstacles between Cockpit and Cabin, Journal of Nanjing University of Aeronautics and Astronautics. 14 (2012) 38-42.

[2] J. Desposito, New antenna allows communications inside a resonant cavity-A crew lock in space, Electronic Design. 46 (1998) 32-33.

[3] R.D. Chute, E.L. Wiener, Safety issues in cockpit/cabin crew communication, Aviation Resource Management. 1 (2000) 205-218.

[4] V.I. Gushin, N.S. Zaprisa, T.B. Kolinitchenko, Content analysis of the crew communication with external communicants under prolonged isolation, Aviation Space and Environmental Medicine. 68 (1997) 1093-1098.

[5] Trine Thorvaldsen, Signe Annie Sonvisen, Multilingual crews on Norwegian fishing vessels: Implications for communication and safety on board, Marine Policy. 43 (2014) 301-306.

[6] Douglas E. Paull, Lori D. Deleeuw, Seth Wolk, The effect of simulation-based crew resource management training on measurable teamwork and communication among interprofessional teams caring for postoperative patients, Journal of Continuing Education in Nursing. 44 (2013) 516-524.

[7] Michael K. Hughes, Ronald S. Benenson, Amy E. Krichten, A Crew Resource Management Program Tailored to Trauma Resuscitation Improves Team Behavior and Communication, Journal of the American College of Surgeons. 219 (2014) 545-551.

[8] A.K. Iusupova, V.I. Gushchin, D.M. Shved, Style of communication between mission control centers and space crews, Aerospace and Environmental Medicine. 45 (2011) 18-24.

[9] Christopher M. Pruitt, Erica L. Liebelt, Enhancing Patient Safety in the Pediatric Emergency Department Teams, Communication, and Lessons From Crew Resource Management, Pediatric Emergency Care. 26 (2010) 942-951.

[10]A. Skogstad, A. Dyregrov, O.H. Hellesoy, Cockpit Cabin Crew Interaction-Satisfaction With Communication and Information Exchange, Aviation Space and Environmental Medicine. 66 (1995) 841-848. 